\title{
The Genetics of Mating Song Evolution Underlying Rapid Speciation: Linking Quantitative Variation to Candidate Genes for Behavioral Isolation
}

\author{
Mingzi $\mathrm{Xu}^{\mathbf{1}}$ and Kerry L. Shaw \\ Department of Neurobiology and Behavior, Cornell University, Ithaca, New York 14853 \\ ORCID IDs: 0000-0001-7947-9138 (M.X.); 0000-0001-8300-0425 (K.L.S.)
}

\begin{abstract}
Differences in mating behaviors evolve early during speciation, eventually contributing to reproductive barriers between species. Knowledge of the genetic and genomic basis of these behaviors is therefore integral to a causal understanding of speciation. Acoustic behaviors are often part of the mating ritual in animal species. The temporal rhythms of mating songs are notably speciesspecific in many vertebrates and arthropods and often underlie assortative mating. Despite discoveries of mutations that disrupt the temporal rhythm of these songs, we know surprisingly little about genes affecting naturally occurring variation in the temporal pattern of singing behavior. In the rapidly speciating Hawaiian cricket genus Laupala, the striking species variation in song rhythms constitutes a behavioral barrier to reproduction between species. Here, we mapped the largest-effect locus underlying interspecific variation in song rhythm between two Laupala species to a narrow genomic region, wherein we find no known candidate genes affecting song temporal rhythm in Drosophila. Whole-genome sequencing, gene prediction, and functional annotation of this region reveal an exciting and promising candidate gene, the putative cyclic nucleotide-gated ion channel-like gene, for natural variation in mating behavior, suggesting that ion channels are important targets of selection on rhythmic signaling during establishment of behavioral isolation and rapid speciation.
\end{abstract}

KEYWORDS Interspecific variation; behavioral barrier; genetic architecture; cyclic nucleotide-gated ion channel; Laupala

C PECIATION can arise from divergence in reproductive phenotypes (Coyne and Orr 2004). Divergent mating behaviors can result in reproductive barriers by causing assortative mating within incipient species. It is well documented that mating behaviors and morphologies diverge early in the speciation process, suggesting an explanation for why prezygotic barriers evolve sooner than postzygotic barriers in the origin of species (e.g., Mendelson 2003; SánchezGuillén et al. 2014). Moreover, some of the most rapid speciation rates known, such as those in Lake Victoria cichlid fish (Seehausen et al. 2008), Hawaiian Laupala crickets (Mendelson and Shaw 2005), Baltic Sea European flounders (Momigliano et al. 2017), and a putative case in Galapagos

Copyright (c) 2019 by the Genetics Society of America

doi: https://doi.org/10.1534/genetics.118.301706

Manuscript received October 16, 2018; accepted for publication January 11, 2019 published Early Online January 15, 2019.

Supplemental material available at Figshare: https://doi.org/10.25386/genetics. 7505762.

${ }^{1}$ Corresponding author: Department of Neurobiology and Behavior, Cornell University, 215 Tower Rd., Ithaca, NY 14853. E-mail: mx52@cornell.edu finches (Lamichhaney et al. 2018), occur when species diverge in mating behaviors and associated structures. Thus, studying the genetics and evolution of behavioral barriers can contribute to an emerging general principle of speciation.

Because evolution is a genetic process, characterizing the genetic architecture and identifying genes involved in behavioral barriers is crucial to understanding targets of selection and establishing causal links among genes, pathways, and mating behaviors in the early stages of speciation. In animals, however, courtship is often complex and multimodal, involving many traits (e.g., Greenspan and Ferveur 2000; Rundus et al. 2010; Starnberger et al. 2014; Ullrich et al. 2016; Mowles et al. 2017). Accordingly, it can be difficult to isolate specific behaviors for genetic analysis. Perhaps because of these complexities, we have a limited understanding of the evolutionary genetics of mating behaviors that contribute to reproductive barriers despite its general importance in speciation.

While some progress has been made in understanding the genetic basis of natural variation in visual and olfactory signals, 
such as cuticular hydrocarbons, sex pheromones, and body coloration [e.g., Gleason et al. 2005, 2009; Kronforst et al. 2006; Sæther et al. 2007; Lassance et al. 2010, 2013; Merrill et al. 2011; Niehuis et al. 2011; Bay et al. 2017; also reviewed by Groot et al. (2016)], many organisms use acoustic signals involving rhythmic neuromuscular behaviors for which we still have a very limited genetic understanding. Even in Drosophila, where acoustic behavior is expressed widely in courtship, we lack a gene-based understanding of natural variation (but see Gleason and Ritchie 2004; Ding et al. 2016). Rhythmic, temporal patterns of such mating "songs" are often species-specific and known components of reproductive barriers among species of insects, fish and amphibians (Gerhardt and Huber 2002; Hartbauer and Römer 2016; Barkan et al. 2017; Smith et al. 2018). The rhythmic elements of song are a result of regularly patterned motor output, products of localized, neural circuits called central pattern generators (CPGs; Chagnaud and Bass 2014; Katz 2016; Schöneich and Hedwig 2017). Compared with other rhythmic mating behaviors such as courtship dance, song rhythms are easy to isolate and measure.

To date, genetic studies of song rhythm variation have revealed a polygenic genetic architecture in insects, including fruit flies, lacewings, crickets, grasshoppers, and moths (Shaw 1996; Williams et al. 2001; Henry et al. 2002; Gleason and Ritchie 2004; Saldamando et al. 2005; Shaw et al. 2007; Ellison et al. 2011; Limousin et al. 2012). However, the causal genes underlying natural variation remain elusive in most cases. Eleven candidate genes that regulate interpulse interval in Drosophila melanogaster, including ion channel genes, transcription factors, and transcription/translation regulators, have been identified through experimentally generated mutations [reviewed by Gleason (2005), also see Turner et al. (2013) and Fedotov et al. (2014, 2018)]. These discoveries offer insight into the types of genes capable of modulating song rhythmicity in naturally occurring systems and thus are reasonable candidate genes for interspecific variation in other singing insects.

Here, we investigate the genetic and genomic basis of natural variation in pulse rate (the inverse of pulse duration, Figure 1) of the male mating song of the endemic Hawaiian cricket Laupala. In Laupala, a rapid radiation has resulted in 38 morphologically and ecologically similar, but acoustically distinctive species (Otte 1994; Mendelson and Shaw 2005). Similar to most crickets, males sing long-range "calling" songs to attract females. Male songs of Laupala are characterized by simple trains of pulses delivered at species-specific rates (Otte 1994; Shaw 2000). Evidence shows that divergent pulse rate partially mediates mate choice (Shaw and Herlihy 2000; Oh and Shaw 2013) and constitutes a reproductive barrier between species (Mendelson and Shaw 2002).

We focus on two closely related species, the slow-calling Laupala paranigra ( 0.71 pulses per second; pps) and the fastcalling Laupala kohalensis (3.72 pps; Shaw et al. 2007; Figure 1), who have diverged roughly 0.43 MYA on the Big Island of Hawaii (Mendelson and Shaw 2005). Roughly 74\% of the difference in pulse rate between these species is due to eight small- to medium-effect, additive QTL (Shaw et al. 2007). In this study, we isolate the largest-effect QTL, examine its inheritance and phenotypic effects, fine-map its location, and test hypotheses about the underlying causal gene or genes. Specifically, we hypothesized that the focal QTL region would include one of several candidate genes for interpulse interval variation in D. melanogaster [Turner et al. 2013; Fedotov et al. 2014, 2018; also reviewed by Gleason (2005)]. Although the specific forms of fly and cricket songs differ, both have species-specific, rhythmic features resulting from regular contractions of thoracic muscles that drive wing movements. Alternatively, the causal gene underlying the focal QTL in our study may be a previously unknown gene. Based on the functional categories of Drosophila candidate genes and biological processes involved in singing, we hypothesize that the pulse rate QTL falls in one of three functional categories: ion transportation, neural modulation and development, and locomotion and muscle development. It may also be the case that regulatory genes affecting the expression of genes belonging to these categories are responsible for the pulse rate variation. We test these hypotheses by conducting whole-genome sequencing (WGS), gene prediction, and functional annotation in the genomic region linked to the focal QTL.

\section{Materials and Methods}

\section{Breeding design}

The breeding design included two stages: isolating the focal QTL (QTL4) in near isogenic lines [NILs; for details see Wiley et al. (2012), Ellison and Shaw (2013) and Supplemental Material, File S1] and generation of NIL mapping populations. We created three replicate NILs (4B, 4C, and 4E) by selectively backcrossing individuals with $L$. paranigra (the slow singer) alleles at the marker linked to QTL4 to L. kohalensis (the fast singer) for four generations (Figure 2). The fourth-generation backcross individuals were then intercrossed to generate NIL lines, where nonrecurrent QTL4 homozygotes were maintained through intercrossing thereafter. Mapping populations were generated by backcrossing one 79th generation NIL male with one L. kohalensis female from the same recurrent line used to generate the NILs, with replication in each NIL (replicates: NIL4B: $n=3$, NIL4C: $n=2$, NIL4E: $n=1$ ). The offspring were intercrossed, resulting in three, two, and one NIL-L. kohalensis backcross $\mathrm{F}_{2}$ (hereafter, $\mathrm{F}_{2}$ ) mapping families for $4 \mathrm{~B}, 4 \mathrm{C}$, and $4 \mathrm{E}$, respectively (denoted as family 4B.1, 4B.2, 4B.3, 4C.5, 4C.9, and 4E.1). We maintained the L. kohalensis recurrent and NIL lines alongside the mapping populations.

All crickets were reared individually in $120 \mathrm{ml}$ specimen cups with a piece of moist tissue and fed ad libitum Organix organic chicken and brown rice dry cat food (Castor \& Pollux Natural Petworks, Clackamas, OR) twice per week in a rearing room held at $20.0^{\circ}$ and a $12: 12 \mathrm{hr}$ light:dark cycle. 

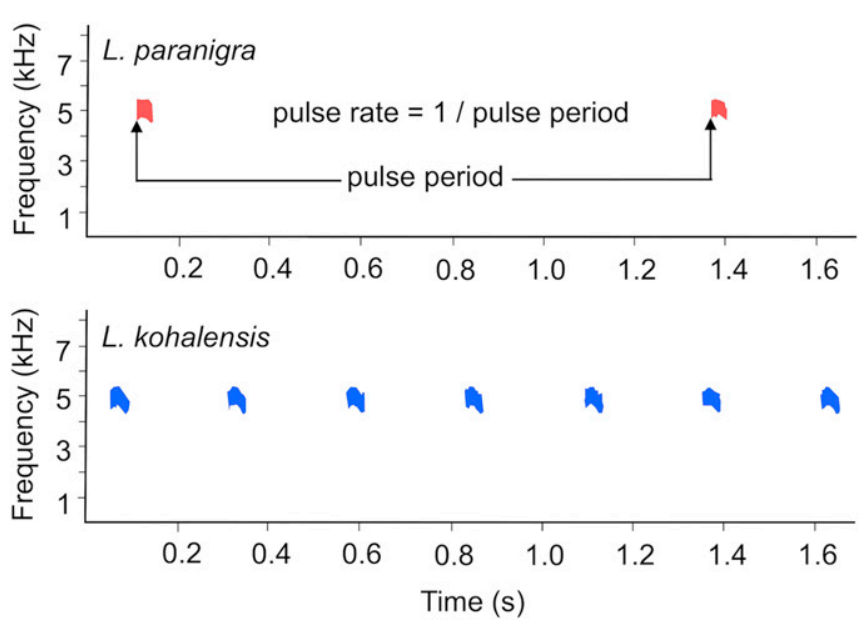

Figure 1 Sonograms of the male calling songs of the slow-calling Laupala paranigra and the fast-calling Laupala kohalensis showing measurement of pulse rate.

\section{Phenotyping}

We recorded male songs with an Olympus WS-852 digital stereo recorder (Olympus Imaging Corp., Tokyo, Japan) during daylight hours in a temperature-controlled room (20.3 \pm $0.01^{\circ}$, mean \pm SE, $n=824$ ). Digital sound files were analyzed using RavenPro 1.4 (http://ravensoundsoftware.com). Pulse period was measured as the time differential between the beginnings of two consecutive pulses (Figure 1). Mean pulse period was calculated from five independent pulse period measurements from a single song bout; mean pulse period was transformed to pulse rate (pps) by taking the inverse of the pulse period.

\section{Genotyping}

We obtained SNP-based genotypes from $\mathrm{F}_{2}$ mapping populations for linkage map estimation and QTL mapping using genotyping-by-sequencing (GBS; Elshire et al. 2011). Sequencing was conducted on the Illumina HiSeq 2000 platform at the Genomic Diversity Facility at Cornell University (see Supplemental File S1 for details of DNA extraction, library preparation, and sequencing). Sequencing reads were demultiplexed with fastq-multx v.1.3.2, base calls at the ends of reads with Phred scrore $<30$ were trimmed, and reads $<50$ bases long were removed with fastq-mcf v.1.04.636 (Aronesty 2011). Processed reads were aligned to the $L$. kohalensis genome reference (NCBI genome assembly ASM231320v1; Blankers et al. 2018a) using Bowtie2 v.2.2.6 (Langmead and Salzberg 2012) with default parameter settings. We called single nucleotide polymorphism (SNP) variants within each $\mathrm{F}_{2}$ family, allowing for a maximum of two mismatches per mapped read using FreeBayes v.0.9.12-2-ga830efd (Garrison and Marth 2012). The resulting SNP markers were filtered using VCFtools 0.1.15 (Danecek et al. 2011) and vcffilter in vcflib v.1.0.0 (Garrison 2012). We retained bi-allelic SNP markers that fulfill the following criteria: (1) $<20 \%$ missing data per family, (2) mi- nor allele frequency $\geq 2.5 \%$, (3) genotype depth $\geq 5$, (4) Phred scaled variant quality $\geq 30$, and (5) strand balance probability for reference and alternative alleles $>0.0001$. Because we did not have the sequences of the parental NIL and L. kohalensis individuals from families 4C.5, 4C.9, and 4E.1, $\mathrm{F}_{2}$ genotypes were called using the $L$. kohalensis genome reference as the $L$. kohalensis parent; the alternative allele was assigned to the NIL parent. For replicate $4 \mathrm{~B}$ where we additionally sequenced parental NIL and L. kohalensis individuals, we further retained SNPs only if the parental L. kohalensis base call was the same as the $L$. kohalensis genome reference and the parental NIL was homozygous for the alternative allele. $\mathrm{F}_{2}$ genotypes for $4 \mathrm{~B}$ families were called using genotypes of the NIL and L. kohalensis grandparents.

Because GBS is a reduced-representation sequencing method that does not reveal contiguous sequence information of a genomic region, we conducted WGS from two male intercross offspring of $\mathrm{BC}_{4}$ in line $4 \mathrm{E}$ (Figure 2). We selected males whose pulse rates suggested that they were homozygous in the introgressed QTL4 region for either L. paranigra or L. kohalensis alleles. The depth of coverage for WGS was roughly $18 \times$ and $12 \times$ for the slow- and fast-singing males, respectively. We identified alternative homozygous SNPs and indels (insertions and deletions) between L. paranigra and L. kohalensis across the introgressed QTL4 region, and used the presence of such SNPs and indels as an indication of increased probability of causation when we evaluated Drosophila song candidate genes and the predicted Laupala genes in this region as the potential causal gene. Paired-end sequencing with an insert size of $200 \mathrm{bp}$ was conducted on the Illumina HiSeq 2000 platform. Parameter values for quality control, read mapping, and variant calling were as used for GBS data. Two sets of SNPs in the final WGS data were of interest: (1) SNPs yielding a homozygous genotype in the fast-calling male that was identical to the reference, and likewise homozygous for the alternative allele in the slow-calling male; and (2) SNPs with no read mapped for the fast-calling male, but with a homozygous alternative genotype (to the reference) for the slow-calling male. SNPs in the first set were filtered with the same criteria as were GBS SNPs except that we allowed no missing genotypes and minor allele frequency $\geq 24 \%$. For SNPs in the second set where no SNP calls could be made for the fast-calling male, the missing data, genotype depth, and alternative variant quality filters were only applied to the slow-calling male. After quality filtering, SNPs in the second set made up $1.4 \%$ of total WGS SNPs. Indels were filtered by the same criteria as SNPs.

\section{Linkage mapping}

Linkage mapping for autosomal linkage groups was conducted with genotypes of both $\mathrm{F}_{2}$ males and females in Joinmap 4 (Van Ooijen 2006), excluding markers that deviated from a segregation ratio of 1:2:1 (Benjamini-Hochberg adjusted $P<0.05$ ) and whose mean depth of coverage was $<20$. When there are multiple markers on the same scaffold, only one marker was retained per $200 \mathrm{~kb}$. Individuals 
1. Near isogenic line (NIL)

2. NIL F2 mapping population

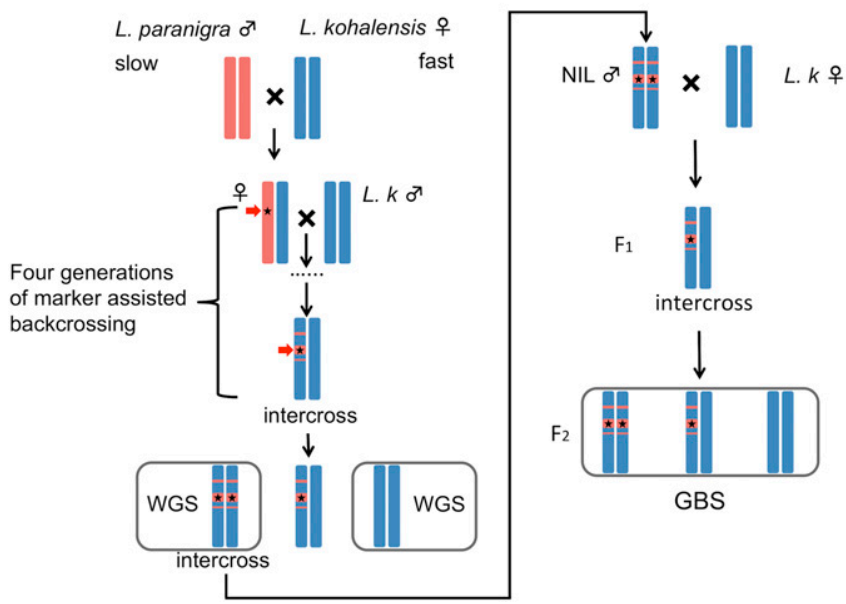

Figure 2 Schematic figure of the two-step breeding design for QTL finemapping of male song pulse rate variation between the slow-calling Laupala paranigra and the fast-calling Laupala kohalensis on linkage group 5 (represented by red and blue bars). In step 1, following the introgression of pure species isofemale lines, near isogenic lines (NILs) were achieved through four generations of marker assisted backcrossing (indicated by the red arrow) selecting for individuals carrying the $L$. paranigra allele at the genetic marker linked to QTL4 in Shaw et al. (2007) (indicated by the black star) and one generation of intercross. Three independent NIL replicates (NIL4B, 4C, and 4E) were established after the intercross. In step 2, seventh to ninth generation NIL males were backcrossed to $L$. kohalensis females to generate segregating $F_{2}$ mapping populations within each NIL replicate. Individuals used for genotypingby-sequencing and whole-genome sequencing were indicated with GBS and WGS, respectively. In replicate $4 \mathrm{~B}$ only, the NIL and L. kohalensis grandparents of the $F_{2}$ mapping populations were also sequenced by GBS (not labeled in the figure).

with $\geq 20 \%$ missing genotype were excluded from linkage mapping.

For each family, markers were grouped using an independent logarithm of the odds (LOD) threshold of 4. Linkage group 5 (LG5) was easily identified by shared markers with previous studies (Blankers et al. 2018a,b). Maps were estimated using the regression algorithm with Kosambi mapping function (details and parameter values are available in File S1).

\section{QTL mapping}

Males with no missing phenotypes and $<20 \%$ missing genotypes on LG5 were used for QTL mapping. We performed standard interval mapping (SIM), two-QTL scan, and multiple QTL mapping (MQM) in each family. MQM was conducted using forward selection with backward elimination. LOD thresholds for SIM were calculated from 20,000 permutations using the maximum likelihood method and LOD thresholds for main and interaction terms in MQM were calculated from 1000 permutations using Haley-Knott regression. We used Haley-Knott regression instead of multiple imputation due to computational constraints. We estimated effect sizes and 1.5-LOD support confidence intervals of significant QTL (see File S1 for further details). All QTL mapping analyses were conducted in R/qtl v.1.39-5 (Broman et al. 2003). Because all two-QTL scan results were consistent with the final MQM results, we only report results from SIM and MQM.

For families where the focal QTL was detected on LG5, we tested whether the phenotypic distribution of $\mathrm{F}_{2}$ males deviates from Mendelian segregation with chi-squared tests. If the primary additive QTL4 has been cleanly isolated, we expect $\mathrm{F}_{2}$ individuals to show 1:2:1 phenotypic segregation. We binned the $\mathrm{F}_{2}$ phenotype data by dividing the range of the phenotypic values evenly into three bins between the maximum and the minimum pulse rate. The Bonferroni-corrected significance level was 0.017 .

\section{Identification of $D$. melanogaster song candidate homologs}

To test the hypothesis that the Laupala QTL 4 region contains one or more homologs of $D$. melanogaster song candidate genes, we first identified homologs of Drosophila song candidate genes in the L. kohalensis genome using a reciprocal blast strategy. We identified 11 genes in Drosophila with experimentally confirmed effects on mean interpulse interval from two sources: a review yielding six genes (Gleason 2005), and a literature search using two combinations of three search terms, including "Drosophila," "courtship song" and "gene" or "Drosophila," "courtship song" and "genetic," from 2005 to present using Google Scholar, yielding five additional genes (Turner et al. 2013; Fedotov et al. 2014, 2018; Table 1). Sequences of these 11 genes were blasted against the L. kohalensis reference genome using tblastx in Blast+ (Camacho et al. 2009), and a new Laupala cerasina transcriptome assembled herein (see File S1) was blasted against the D. melanogaster protein database using tblastn at an E-value cutoff of 10

We then obtained the conserved domains of these genes from the conserved domain database at NCBI (MarchlerBauer et al. 2015) using default settings and confirmed the detected domains using the HomoloGene database at NCBI (Table S1). We inferred homology between L. kohalensis sequence and a described D. melonagaster gene if (1) the $L$. kohalensis and the $D$. melanogaster genes were mutual best blast hits in the reciprocal blast, (2) the matched sequences were in synteny, and (3) at least half of the conserved domains of a given $D$. melanogaster gene contain blast hits matching the $L$. kohalensis query. In cases where the best blast hit in one direction is not the best hit in the other direction but was within the top five best matches, we designated the sequence with the longest match, the most conserved domains and with complete synteny as the Laupala homolog.

We evaluated the likelihood of putative Drosophila homologs as the causal gene underlying the focal QTL by two criteria: (1) its hosting scaffold resides within the 1.5-LOD confidence interval of QTL4, and if so, (2) the gene and the putative regulatory region $20 \mathrm{~kb}$ up- and downstream from the gene contain at least one WGS SNP or indel between the alternative homozygote males from line 4E. To decrease the 
Table 1 Experimentally verified candidate genes in Drosophila melanogaster that affect mean interpulse interval of the courtship song, the location and linkage group assignment of their putative homologs in the Laupala kohalensis genome, and homology and synteny between the Drosophila candidate genes and putative Laupala homologs

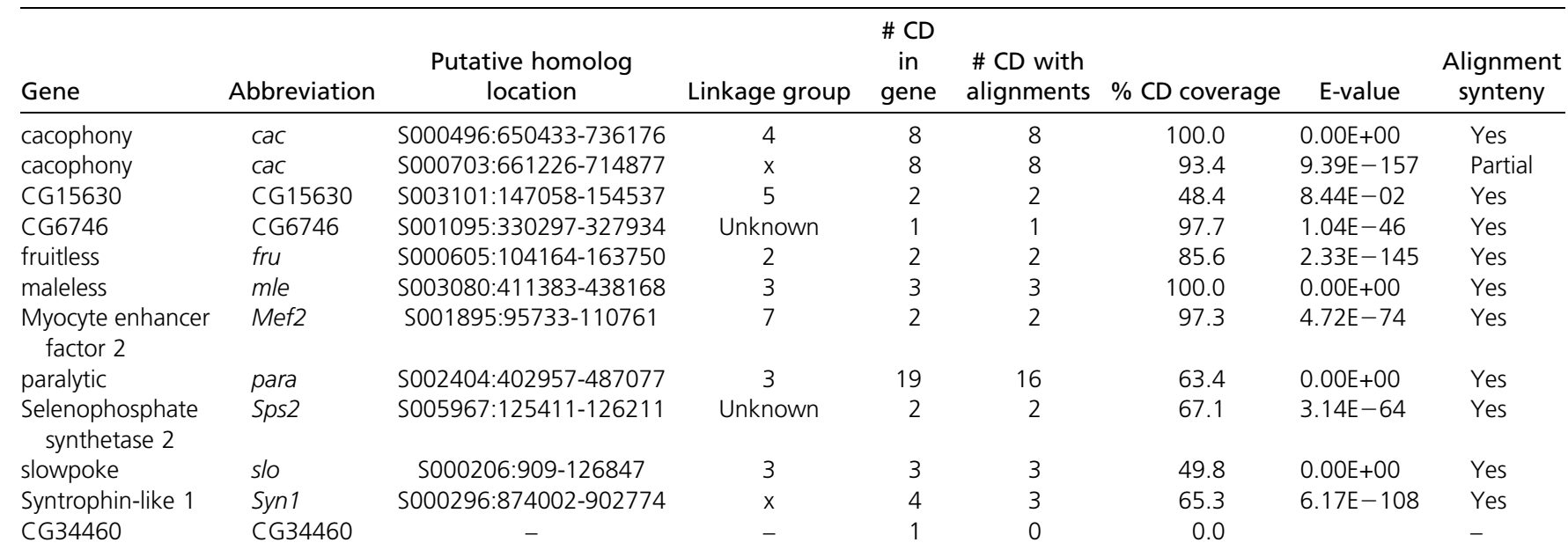

The number of conserved domains (CDs), the proportion of total CD length covered by significant alignments, the $\mathrm{E}$ value and alignment synteny were from a tblastx between the $D$. melanogaster candidate genes and the $L$. kohalensis genome

potential for a false negative, we used a $20 \mathrm{~kb}$ up- and downstream region as the size of the putative regulatory region, rather than the conventional $5 \mathrm{~kb}$ window. We assigned scaffolds hosting Drosophila homologs to Laupala linkage groups estimated in this and another study where linkage maps of three intercrosses of Laupala species pairs were constructed (Blankers et al. 2018a). We then examined whether any scaffold hosting a putative Drosophila homolog resides within the 1.5-LOD confidence intervals of the major QTL on LG5. Putative homologs that could not be assigned to a linkage group (i.e., their hosting scaffolds are not in any available linkage maps) were evaluated solely on the basis of whether they contained WGS SNPs or indels.

Lastly, to investigate if any scaffold within the confidence interval of QTL4 contains divergent paralogs of the Drosophila candidate genes that escaped detection by the method above, we blasted the sequences of conserved domains in the Drosophila candidate genes against scaffolds within the confidence interval of the QTL using an E-value cutoff of $1 \mathrm{E}-5$. A diverged paralog would be expected to contain at least one significant blast hit in the conserved domains. Conversely, failure to see a significant blast hit indicates the absence of orthologs or paralogs of Drosophila candidate genes.

\section{Gene prediction and functional annotation}

To identify L. kohalensis candidate genes causing pulse rate variation on LG5, we annotated scaffolds within and flanking the 1.5-LOD confidence interval of the major QTL in 4C.9 (the most robust QTL identified and the family with the highest sample size). We chose the 1.5-LOD confidence interval by convention, yet because the LOD profile of the final QTL model drops sharply on both sides of the major QTL peak, the scaffolds included in the 1.5-LOD confidence interval are identical to those up to a 5-LOD confidence interval. We therefore consider these scaffolds sufficiently inclusive for our candidate gene search. Gene prediction was done using the Maker pipeline (Cantarel et al. 2008) with available RNA, EST, and protein evidence (Danley et al. 2007; Bailey et al. 2013; Zeng et al. 2013; Berdan et al. 2016). Detailed information on gene prediction can be found in the File S1. Briefly, we performed two rounds of training with SNAP (Korf 2004) and Augustus-3.2.3 (Stanke and Morgenstern 2005) with the 10 longest scaffolds and the five focal scaffolds in the $L$. kohalensis reference genome in a bootstrap manner to obtain Laupala-specific gene model predictions. The second-round gene model outputs from both SNAP and Augustus were used to predict gene structures on the five focal scaffolds in the third round.

To examine predicted genes, we performed functional annotations in Blast2GO 4.1 (Götz et al. 2008). Specifically, we blasted sequences of all predicted genes against the NCBI nonredundant protein database using an E-value cutoff of $1 \mathrm{E}-4$ as well as InterPro (Finn et al. 2016) with the default setting, and inferred the potential gene identity with the automatic annotation description function and manual curation. We then annotated gene ontologies (Ashburner et al. 2000) for predicted genes using NCBI and InterPro blast results as well as UniProt (Apweiler et al. 2004) and KEGG databases (Kanehisa et al. 2016) with default parameter values in Blast2GO. To interrogate further the identities of any remaining predicted genes lacking an annotation, we reran blast for these sequences using a relaxed E-value cutoff of 100 .

To further implicate candidate genes as the cause of pulse rate variation, we tallied WGS SNPs and indels in the QTL4 region (within annotated genes and $5 \mathrm{~kb}$ up- and downstream) from the two alternative homozygous 4E males using SnpEff (Cingolani et al. 2012) and manually, respectively. We evaluated the possibility of a causal role for a given predicted 
gene in pulse rate variation by three criteria: (1) the gene maps to a scaffold within the 1.5-LOD confidence interval, (2) the gene has at least one WGS SNP or indel within the coding or (putative) regulatory region, and (3) orthologs or paralogs of the gene play a functional role in rhythmic movements in other organisms. For the most promising candidate gene fulfilling all three criteria, we confirm the identity of the predicted gene by both a more focused sequence alignment using protein products of representative members of the indicated gene family in Exonerate 2.2.0 (Slater and Birney 2005) and phylogenetic inference using the amino acid sequences of the conserved domains in these proteins in PhyML 3.0 (Guindon and Gascuel 2003). We further determined WGS SNPs and indels as regulatory, intronic, synonymous, or nonsynonymous. For details of the gene identity inference and SNP/indel effect annotation, see File $\mathrm{S} 1$.

\section{Data availability}

All DNA and RNA sequences, the L. cerasina transcriptome, and the associated metadata are available at NCBI (DNA and RNA sequences: PRJNA509479; transcriptome: GHDK00000000). Phenotypic data, SNP genotypes, bioinformatic and QTL mapping scripts, and metadata (in the readme file) are available for download at https://github.com/ MingziXu/QTL4-male-fine-mapping-scripts. File S1 contains additional method details and supplemental results; Table S1 contains information about conserved domains in the $11 \mathrm{D}$. melanogaster candidate genes for variation in interpulse interval of courtship song; Table S2 contains summary statistics of the linkage maps; Table S3 contains results from MQM on linkage groups other than LG5; Table S4 contains frequency distributions of the $\mathrm{F}_{2}$ male pulse rates and chi-squared test results for Mendelian segregation ratios in $4 \mathrm{C}$ and $4 \mathrm{E}$ families; Table S5 shows results of functional annotation of the 66 predicted genes on the five focal scaffolds within and flanking the 1.5-LOD (same as 5-LOD) support confidence interval of the major QTL peak in 4C.9; Table S6 contains information about conserved domains in CNG and HCN channels used for protein tree construction; Table S7 contains descriptions of top blast hits and the E-values for unannotated genes in Table S5 from a blast using relaxed E-value cutoff of 100; Figure S1 shows the phenotypic distribution in NIL4B; Figure S2 shows homology and synteny of linkage groups among six $\mathrm{F}_{2}$ families; Figure S3 shows the linkage maps of (a) NIL4C and NIL4E and (b) NIL4B; Figure S4 shows the LOD profile of MQM and SIM models in NIL4B; Figure S5 shows synteny between the linkage map of 18 markers showing potential double recombination patterns on LG5 and the linkage map for LG5 used for QTL mapping in 4C.9; Figure S6 shows alignment between protein sequence of the termite Zootermopsis nevadensis cyclic nucleotide-gated olfactory channel-like protein and the putative Laupala Cngl homolog. Supplemental material available at Figshare: https://doi.org/ 10.25386/genetics. 7505762 .

\section{Results}

\section{Phenotypic distribution of L. kohalensis line and NILS}

Males from the parental $L$. kohalensis line showed expected $L$. kohalensis pulse rates $(n=24,3.80 \pm 0.11 \mathrm{pps}$, mean \pm SD) and males from the three replicates of NIL showed expected slower pulse rates by substituting the $L$. paranigra allele at QTL4 (Figure 3 and Figure S1; NIL4B: $n=4,3.12 \pm 0.13$ pps; NIL4C: $n=7,3.07 \pm 0.05$ pps; NIL4E: $n=7,2.95 \pm$ 0.04 pps).

\section{Linkage mapping}

The linkage maps of the six families contained seven autosomal linkage groups where marker orders were highly consistent (Figure 4 and Figure S2). Among all linkage groups, LG5 had the highest number of shared markers among replicates (Figure S2 and Figure S3). Family 4C.9 had the highest number of markers and the highest marker density of all families (Figure 4 and Table S2).

Families 4C.5, 4C.9 and 4E.1 shared a large and dense group of markers in the central part of LG5 that was absent in 4B families (Figure 4, red). We discuss results from 4C/4E and $4 \mathrm{~B}$ families separately (see below).

\section{QTL mapping in NIL4C and $4 E$}

Using SIM, we estimated the location of a large effect ( $\sim 87.25 \%$ of $\mathrm{F}_{2}$ variance) QTL segregating in 4C.9 on LG5. With MQM, we additionally estimated the position of a smaller effect QTL ( $\sim 1 \%$ of $\mathrm{F}_{2}$ variance) on LG5 (Figure 5 and Table 2). Together, these two QTL explain $88.3 \%$ of the phenotypic variance for pulse rate in 4C.9; no other QTL were detected in this family. Similarly, both a major QTL, identified with both SIM and MQM, and a minor QTL, identified using MQM, were localized on LG5 in 4C.5 (Figure 5 and Table 2), which together explain $87.8 \%$ of the variation segregating for pulse rate in this $\mathrm{F}_{2}$ mapping population (Table 2). In 4C.5, we also detected four additional, small-effect QTL on LG1, LG2, LG3, and LG7 (Table S3). Lastly, we detected a single QTL on LG5 in 4E.1 using SIM and MQM, comparable in size ( $\sim 81 \%$ of segregating $\mathrm{F}_{2}$ variance) to those identified in 4C.9 and 4C.5. Several smaller-effect QTL were also identified in 4E.1, localized to LG1, LG3, LG4, and LG7 (Table S3). Concomitant with the introgression pattern revealed by QTL mapping, $\mathrm{F}_{2}$ generation males from $4 \mathrm{C}$ and $4 \mathrm{E}$ families each exhibited the classical Mendelian 1:2:1 phenotypic segregation pattern for pulse rate (Figure 3 and Table S4). Hereafter, we refer to the large-effect QTL on LG5 identified in 4C.9, 4C.5, and 4E.1 as the "major" QTL.

The locations of the major QTL on 4C.5, 4C.9, and 4E.1 are highly consistent with each other, as in each family the same SNP (at base location 224,758 on scaffold S000353) is the marker that exhibits the highest LOD score by SIM. In MQM, the marker on scaffold S000353 has the highest LOD score in 4C.9 and 4E.1; the SNP at base location 297,109 on scaffold S001839, the scaffold immediately adjacent to S000353, has 

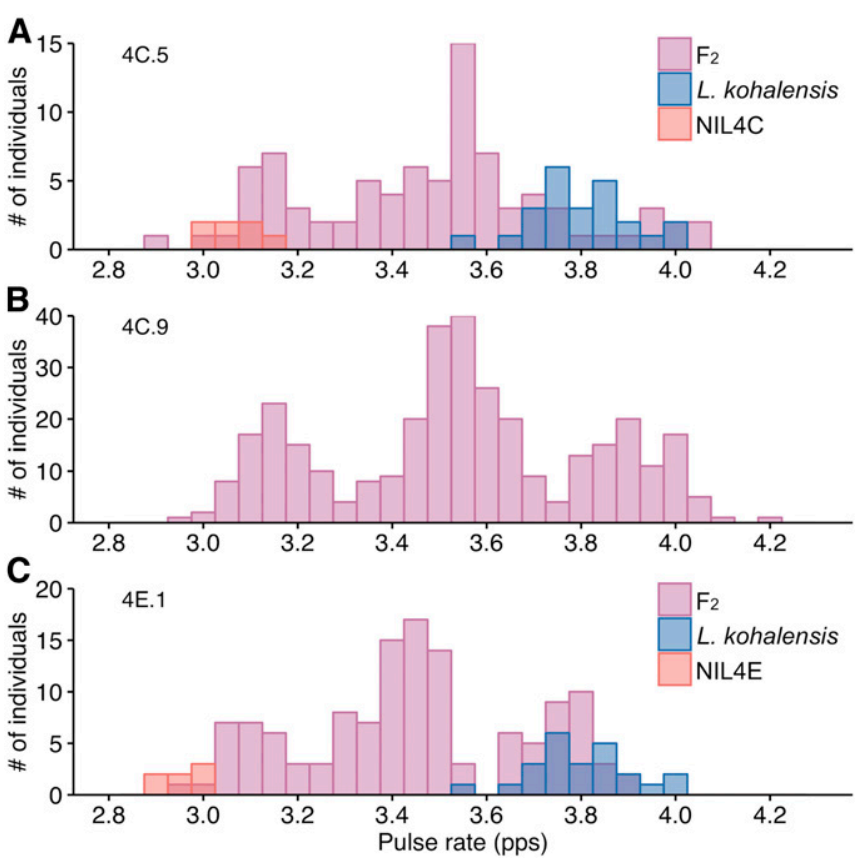

Figure 3 Phenotypic distribution of $F_{2}$ males in (A) 4C.5, (B) 4C.9, and (C) $4 \mathrm{E}$.1. The phenotypic distributions of parental $L$. kohalensis and NIL individuals are shown in $(A)$ and $(C)$.

the highest LOD score in 4C.5 (Table 3). Further, the 1.5-LOD confidence intervals of the major QTL are narrow and largely overlap in 4C and 4E families (Figure 5 and Table 3), containing seven (including five mapping to the same position), five (including two mapping to the same position), and two scaffolds in 4C.5, 4C.9, and 4E.1, respectively.

The major QTL in 4C and 4E families also have similar phenotypic effects (Table 2). In all three families, the phenotypic distributions at the markers with the highest LOD scores showed the expected slow pulse rates for the L. paranigra genotype (AA, Figure 5, 4C.5: $3.18 \pm 0.02$, 4C.9: $3.17 \pm$ $0.01,4 \mathrm{E} .1: 3.12 \pm 0.01 \mathrm{pps}$ ), fast pulse rates for the L. kohalensis genotype (BB, 4C.5: $3.86 \pm 0.03$, 4C.9: $3.90 \pm 0.01$, 4E.1: $3.75 \pm 0.02 \mathrm{pps}$ ), and intermediate pulse rates for heterozygotes (4C.5: $3.52 \pm 0.02$, 4C.9: $3.54 \pm 0.01$, 4E.1: $3.43 \pm 0.01 \mathrm{pps})$. There was little phenotypic overlap between the genotypes (Figure 5). The phenotypic effect of a single allele at the major QTL on LG5 was predominantly additive in $4 \mathrm{C}$ and $4 \mathrm{E}$ families, contributing $10-12 \%$ of the phenotypic difference between the original parent ( $L$. kohalensis and L. paranigra) phenotypes (Table 2).

\section{QTL mapping in NIL4B}

In both 4B.1 and 4B.2, we detected a minor effect QTL in the beginning of LG5 (Figure S4 and Table 2), but no QTL were detected in the central part of the linkage group near the major QTL found in 4C and 4E families. The introgressed region in $4 \mathrm{~B}$ families did not include markers within the 1.5-LOD confidence intervals of the major QTL in $4 \mathrm{C}$ and 4E families (Figure 4 and Figure 5) and all markers within the confidence interval of $4 \mathrm{C}$ and $4 \mathrm{E}$ were homozygous for $L$.

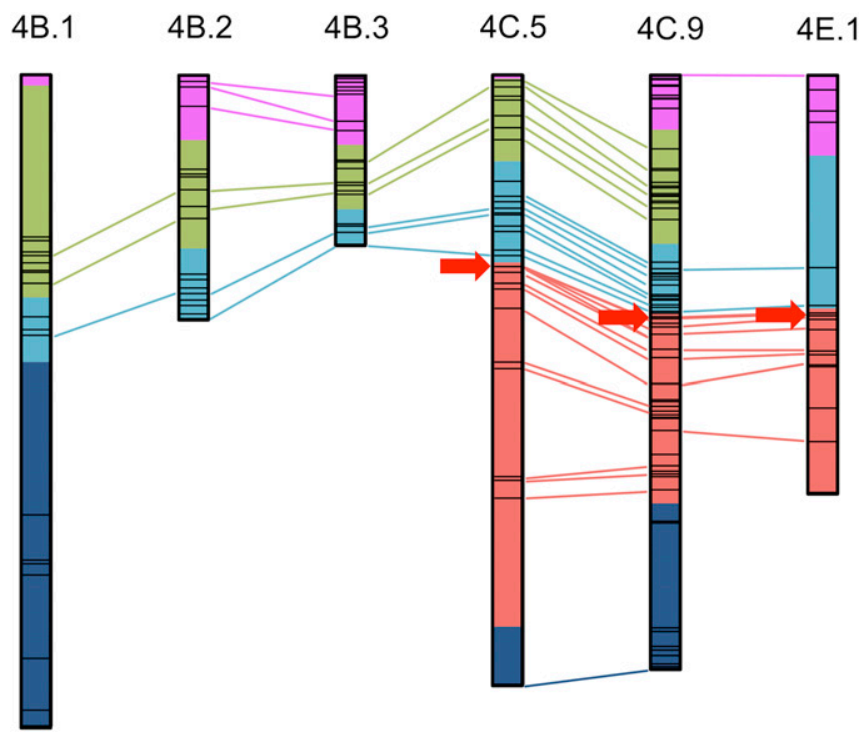

Figure 4 Linkage maps of linkage group 5 in six $F_{2}$ mapping families. Different clusters of markers based on their presence and absence in different families are color coded and markers shared between two families are connected with colored lines. The red arrows indicate the positions of the major QTL from the final multiple QTL models. Linkage maps with marker names can be found in Figure S3.

kohalensis genotypes in 4B families. No QTL were detected on LG5 in 4B.3. We also detected several moderate-effect and primarily additive QTL on LG1 and LG7 in 4B families (Table S3).

\section{Putative homologs of D. melanogaster song candidate genes}

Reciprocal blast identified putative homologs for 10 out of 11 candidate genes in the L. kohalensis genome (Table 1 ). These genes had several identifiable conserved domains with high coverage and extensive synteny across matched regions, and in all cases, we identified transcripts that mapped to these genes in the Laupala transcriptome. In contrast, CG34460 (Fedotov et al. 2014) had no significant match in the $L$. kohalensis genome to the conserved domain region, nor a Laupala transcript match, at an E-value cutoff of 10.

Putative Laupala homologs of 10 Drosophila genes were distributed across six different linkage groups (Table 1). Only one putative homolog (CG15630) groups with LG5 in Laupala. However, the scaffold hosting the putative homolog locates between scaffold S001650 and S000820, outside the confidence intervals of both the major and the minor QTL on LG5 (Figure S5 and Table 3). We were unable to assign the putative homologs of CG6746 and Sps2, residing on scaffolds S001095 and S005967, respectively, to any linkage group. However, we detected no alternative homozygous WGS SNPs or indels between parental lines within the putative gene or the extended $20 \mathrm{~kb}$ putative regulatory regions for these two homologs. Moreover, no scaffold within the confidence interval of the major or minor QTL on LG5 contained significant blast hits to conserved domains of $D$. melanogaster candidate genes. 

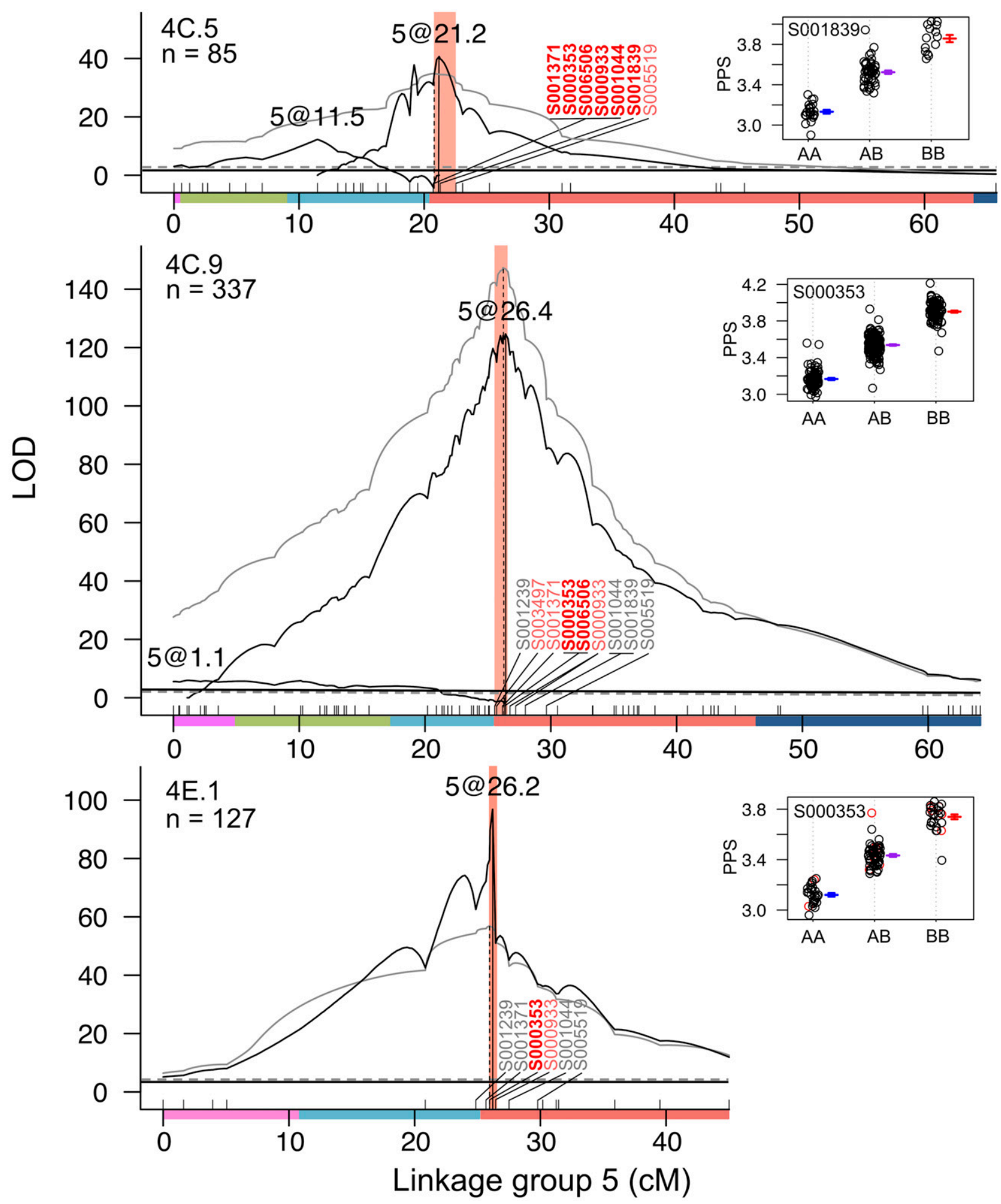

Figure 5 LOD profiles of the final multiple QTL mapping models (MQM, shown in black) and standard interval mapping models (SIM, shown in gray) for 4C and 4E families. The shaded areas indicate 1.5-LOD support confidence intervals, the vertical lines indicate the locations of the peaks and the horizontal lines indicate the significance thresholds for QTL; solid and dashed lines are from MQM and SIM, respectively. Clusters of markers on the linkage group are represented by colored bars on the linkage maps and the same color scheme from Figure 4 is used. Markers within and flanking the 1.5-LOD support confidence intervals are labeled in red, markers with the highest LOD scores from the MQM and SIM are in bold, and markers shared among families but are outside the 1.5-LOD confidence intervals are labeled in gray. The top right panels show phenotypes of $F_{2}$ males at the SNP marker with the highest LOD score from the MQM. "A" denotes $L$. paranigra genotype and " $B$ " denotes $L$. kohalensis genotype. Open black circles represent an individual with existing genotype and open red circles represent an individual with imputed genotype. The colored horizontal lines and error bars represent mean \pm SE. 
Table 2 Standard interval mapping and multiple QTL mapping results on linkage group 5 for five $F_{2}$ mapping populations showing the location, LOD score, 1.5-LOD support confidence interval, and three measures of the phenotypic effect for each QTL

\begin{tabular}{|c|c|c|c|c|c|c|c|c|c|c|c|c|}
\hline \multirow[b]{2}{*}{$\begin{array}{l}\text { Linkage } \\
\text { group }\end{array}$} & \multirow[b]{2}{*}{$\begin{array}{l}\text { QTL } \\
\text { class }\end{array}$} & \multirow[b]{2}{*}{ Family } & \multirow[b]{2}{*}{$\begin{array}{l}\text { Sample } \\
\text { size }\end{array}$} & \multicolumn{3}{|c|}{ Standard interval mapping } & \multicolumn{3}{|c|}{ Multiple QTL mapping } & \multirow[b]{2}{*}{$\begin{array}{l}\text { Additive } \\
\text { effect size } \\
\text { (pps) }\end{array}$} & \multirow[b]{2}{*}{$\begin{array}{l}\% \text { Species } \\
\text { difference } \\
\text { explained }\end{array}$} & \multirow[b]{2}{*}{$\begin{array}{c}\% \mathrm{~F}_{2} \\
\text { variance } \\
\text { explained }\end{array}$} \\
\hline & & & & $\begin{array}{c}\text { QTL } \\
\text { location } \\
\text { (cM) }\end{array}$ & $\begin{array}{l}\text { LOD score } \\
\text { (LOD } \\
\text { threshold) }\end{array}$ & $\begin{array}{l}\text { 1.5-LOD } \\
\mathrm{Cl}(\mathrm{cM})\end{array}$ & $\begin{array}{c}\text { QTL } \\
\text { location } \\
\text { (cM) }\end{array}$ & $\begin{array}{l}\text { LOD score } \\
\quad \text { (LOD } \\
\text { threshold) }\end{array}$ & $\begin{array}{l}\text { 1.5-LOD } \\
\mathrm{Cl}(\mathrm{cM})\end{array}$ & & & \\
\hline 5 & Major & $4 C .9$ & 337 & 26.20 & $147.36(3.23)$ & $25.80-26.75$ & 26.40 & $124.64(3.14)$ & $25.80-26.60$ & $0.34 \pm 0.01$ & 11.30 & 87.25 \\
\hline 5 & Major & 4E.1 & 127 & 25.96 & $56.88(3.15)$ & $24.86-26.40$ & 26.20 & $96.83(2.84)$ & $25.96-26.40$ & $0.32 \pm 0.01$ & 10.63 & 81.17 \\
\hline 5 & Major & $4 C .5$ & 85 & 20.70 & $34.85(3.30)$ & $19.60-22.54$ & 21.20 & $40.67(3.09)$ & $20.80-21.60$ & $0.35 \pm 0.02$ & 11.63 & 86.07 \\
\hline 5 & Minor & $4 C .9$ & 337 & - & - & - & 1.09 & $5.97(3.14)$ & $0.00-11.65$ & $0.04 \pm 0.01$ & 1.33 & 1.01 \\
\hline 5 & Minor & 4B.2 & 63 & - & - & - & 15.00 & $4.15(3.23)$ & $0.00-26.35$ & $0.11 \pm 0.02$ & 3.65 & 21.62 \\
\hline
\end{tabular}

Note that the proportion of phenotypic difference between the two parental species explained by a single allele is capped at $50 \%$. QTL effects were estimated from the multiple QTL models. 4B.3 does not have any significant QTL, hence, was not shown in the table.

\section{Annotation of the QTL region}

The 1.5-LOD confidence interval of the major QTL on LG5 contained five scaffolds in 4C.9 (Table 3). Gene predictions resulted in a total of 66 genes on four of the five scaffolds (Table S5). One scaffold (S006506, $51.9 \mathrm{~kb}$ total length) contained no predicted gene, had no mapped transcript or EST, and had no blast hit against any protein databases. Among the 66 predicted genes, 38 had significant blast hits against the nonredundant protein database, 21 of which had gene ontology annotations (Table S5). The annotated genes have functions pertaining metabolism and biosynthesis, cell cycle regulation, cell differentiation, DNA repair, immune response, development, cell signaling, and transmembrane transportation. Among these functional categories, three are potentially relevant to pulse rate variation: ion transmembrane transportation (Table S5, genes \#20, \#22, \#26), synaptic vesicle transportation (gene \#23), and muscle development and locomotion (gene \#26). All these genes locate on scaffolds with the top two LOD scores.

\section{Evaluation of the predicted genes}

We further evaluated the likelihood of annotated genes as the causal gene underlying pulse rate variation using WGS SNPs and indels. The two males used for WGS had song pulse rates of 3.69 and 2.98 pps, respectively. Between these two males, we identified a total of 1664 SNPs, distributed among four of the five scaffolds (S006506 contains no WGS SNP) within the major QTL confidence interval. Among 1664 SNPs, 416 located within 28 (of the 66) predicted genes or in the putative regulatory regions (Table S5). A single-nucleotide deletion and a single-nucleotide insertion were found on scaffolds S000933 and S003497, respectively, both of which intergenic and neither was in the putative regulatory region.

Among 38 predicted genes with annotations, only one gene (Table S5, gene \#20) fulfilled all three criteria for potential causal genes. The gene between base locations 312,523 and 384,104 on scaffold S001371 (with the second highest LOD score; Table 3) matches cyclic nucleotide-gated ion channellike gene $(\mathrm{Cngl})$, whose protein product is a distinct member of the cyclic nucleotide-gated ion channel (CNG) family (Figure 6) that contains ion channels gated by cAMP or cGMP. Closely related ion channels gated by cyclic nucleotides are known to be involved in rhythm generation, e.g., leech heartbeat, mammalian locomotion, and respiratory networks (Thoby-Brisson et al. 2000; Yamada et al. 2005; Herrmann et al. 2015; Calabrese et al. 2016; Zhu et al. 2016). Among the putative Cngl homologs we identified among animals (Table S6), the putative Laupala Cngl is most similar to the cyclic nucleotide-gated olfactory channel-like gene in termites (Figure 6, Figure 7 and Figure S6), then to AgaP AGAP003349-PB in mosquitos, and then to Cngl in D. melanogaster (Figure 6, Figure 7).

\section{Discussion}

Dissecting the genetic causes of variation underlying the evolution of reproductive barriers is integral to understanding speciation (Coyne and Orr 2004; Shaw and Mullen 2011). Mating behaviors can be powerful "speciation phenotypes" because their divergence is an effective means to curtail gene flow between incipient species and maintain reproductive boundaries. Moreover, divergence in mating behaviors often coincides with the most rapid speciation events (Mendelson and Shaw 2005; Seehausen et al. 2008; Momigliano et al. 2017; Lamichhaney et al. 2018). Yet evidence linking genetic variation in specific mating behaviors to reproductive boundaries in nature is rare.

The Hawaiian cricket Laupala has experienced extremely rapid speciation, with the most closely related species showing distinctive song differences, supporting a role for acoustic behavior in the origin of species (Otte 1994; Mendelson and Shaw 2005). The species-specific acoustic signals of Laupala males and corresponding female preferences have been found to contribute to reproductive barriers between species (Mendelson and Shaw 2002; Oh et al. 2013). Because of the ease of generating laboratory hybrids and isolating specific mating behaviors, Laupala crickets are a promising 
Table 3 Names, map positions, LOD scores, and types of markers within and flanking the 1.5-LOD confidence intervals of the major QTL from multiple QTL mapping models in $4 \mathrm{C}$ and $4 \mathrm{E}$ families

\begin{tabular}{llcrl}
\hline & & & LOD & \\
Family & Marker name & Map position (cM) & score & Marker Type \\
\hline 4C.5 & S001371_680831 & 20.724 & 30.98 & Flanking \\
4C.5 & S000353_224758 & 20.724 & 30.98 & Flanking \\
4C.5 & S006506_7146 & 20.724 & 30.98 & Flanking \\
4C.5 & S000933_697595 & 20.724 & 30.98 & Flanking \\
4C.5 & S001044_55790 & 20.724 & 30.98 & Flanking \\
4C.5 & 5@21.2 & 21.200 & 40.67 & Peak \\
4C.5 & S001839_297109 & 21.282 & 39.86 & Cl \\
4C.5 & S005519_323363 & 22.543 & 27.10 & Flanking \\
4C.9 & S003497_180469 & 25.692 & 115.01 & Flanking \\
4C.9 & S001371_680831 & 26.147 & 121.44 & Cl \\
4C.9 & S000353_224758 & 26.298 & 122.88 & Cl \\
4C.9 & S006506_7146 & 26.298 & 122.88 & Cl \\
4C.9 & 5@26.4 & 26.400 & 124.65 & Peak \\
4C.9 & S000933_1324863 & 26.754 & 117.47 & Flanking \\
4E.1 & S001239_386983 & 24.865 & 62.52 & Flanking \\
4E.1 & S001371_680831 & 25.956 & 72.17 & Flanking \\
4E.1 & S000353_224758 & 25.956 & 79.52 & Flanking \\
4E.1 & 5@26.2 & 26.200 & 96.83 & Peak \\
4E.1 & S000933_697595 & 26.440 & 50.99 & Flanking \\
\hline
\end{tabular}

Markers whose names start with "S" are SNPs and markers whose names start with "5@" are phantom markers from simulations in multiple QTL models. Marker type is categorized as peak (the marker closest to the QTL peak location), $\mathrm{Cl}$ (markers within the confidence interval), and flanking (markers immediately outside the confidence interval boundaries on both sides). Because the LOD profile in $4 \mathrm{E} .1$ drops much more sharply on the left side of the peak than on the right side, we also included two additional markers outside the left boundary of the confidence interval that have higher LOD score than the immediate right flanking marker.

system to identify causal genes underlying behavioral barriers and speciation. Here, we (1) isolate the largest-effect QTL for a pulse rate difference between $L$. kohalensis and $L$. paranigra through selective introgression, (2) fine-map the focal QTL and produce a physical map of the QTL region, (3) test if any Drosophila candidate genes for song interpulse interval variation map to the focal QTL region in Laupala, and (4) identify strong candidate genes underlying the focal QTL.

Multiple lines of evidence show that we successfully moved the largest-effect QTL from the slow pulsing species (L. paranigra) into the genomic background of the fast-pulsing species (L. kohalensis), isolating variation at this locus from the polygenic background of pulse rate differences. First, pooling across all families, we found more and a higher density of markers on LG5 than on other linkage groups (Table S2), consistent with selective introgression of QTL4 into NILs. Second, we identified a major QTL on LG5 in 4C and 4E families that explained nearly all $\mathrm{F}_{2}$ phenotypic variation (>80\%; Table 2) and exhibited iconic 1:2:1 Mendalian segregation (Figure 3 and Table S4). Although additional QTL were identified in 4C.5 and 4E.1, each effect was minor $(<\sim 5 \%)$ in comparison with the major QTL (Table S3), suggesting successful isolation of a single major locus in replicates $4 \mathrm{C}$ and $4 \mathrm{E}$. Third, the major QTL peak in $4 \mathrm{C}$ and $4 \mathrm{E}$ families are linked to the same SNP marker, with similar estimated phenotypic effect sizes, suggesting that the major QTL in 4C.5, 4C.9, and 4E.1 are the same locus. We also note that the effect size of the major QTL in each family (4C.5, 4C.9, and 4E.1) explains roughly $10 \%$ of the species difference, similar to a previous estimate on this linkage group in an $\mathrm{F}_{2}$ mapping study using AFLP markers (Shaw et al. 2007). Thus, the major QTL in 4C.5, 4C.9, and 4E.1 and QTL4 in the previous mapping study (Shaw et al. 2007) appear to be the same locus.

Phenotypic data from replicates $4 \mathrm{C}$ and $4 \mathrm{E}$ exhibited the classical 1:2:1 Mendelian segregation pattern (Figure 5 and Table S4), demonstrating that the major QTL contributing to natural variation of behavioral divergence follows a simple Mendelian segregation rule. Our result here suggests that the genetic underpinning of a complex behavioral trait can be understood element by element with simple Mendelian inheritance rules.

Mapping results testified that we have substantially improved resolution, power, and precision in localizing the major locus. Previous efforts with AFLP markers produced an average marker spacing between 5 and $8 \mathrm{cM}$ (Shaw et al. 2007). In comparison, we achieved a median marker spacing of $1.67 \mathrm{cM}$ for all linkage groups and $0.80 \mathrm{cM}$ for LG5 (Table S2), increasing the map resolution by roughly 10-fold on LG5. Moreover, LOD scores and confidence intervals associated with the focal QTL on LG5 were vastly improved, and the distance between the QTL peaks and the closest markers have decreased from 3-5 to $0.1-0.2 \mathrm{cM}$ in the current study (Table 3).

In contrast to results in $4 \mathrm{C}$ and $4 \mathrm{E}$ families, there were no segregating markers within the confidence interval region of the major QTL in any of the three 4B families (all were homozygous for the L. kohalensis genotype). Concomitantly, no major QTL were detected in any 4B family. The likely explanation is that recombination may have occurred between the L. paranigra markers used for selective backcrossing and QTL4. Linkage mapping data and genotypes of NIL4B individuals suggested a breakpoint between QTL4 and scaffold S001239, the first scaffold immediately outside the confidence interval of the major QTL (Figure S3A). Replicate 4B families thus serve as a (accidental) negative control, by exhibiting both the lack of a major QTL and any segregating markers within the confidence interval of the major QTL. The observed phenotypic segregation in $\mathrm{F}_{2}$ individuals in 4B was thus likely due to effects of QTL on LG1, LG7, and other minor QTL that may not have been detected in the QTL models. The lack of the 1:2:1 phenotypic segregation pattern in $4 \mathrm{~B}$ families is consistent with this explanation.

We found no evidence to support the hypothesis that the major QTL on LG5 is homologous to a candidate gene for variation in interpulse interval of courtship song in Drosophila. None of the putative Drosophila homologs we localized in the Laupala genome map within the confidence intervals of either the major or the minor QTL on LG5 (Table 1). The two putative homologs for genes CG6746 and Sps2 to which 


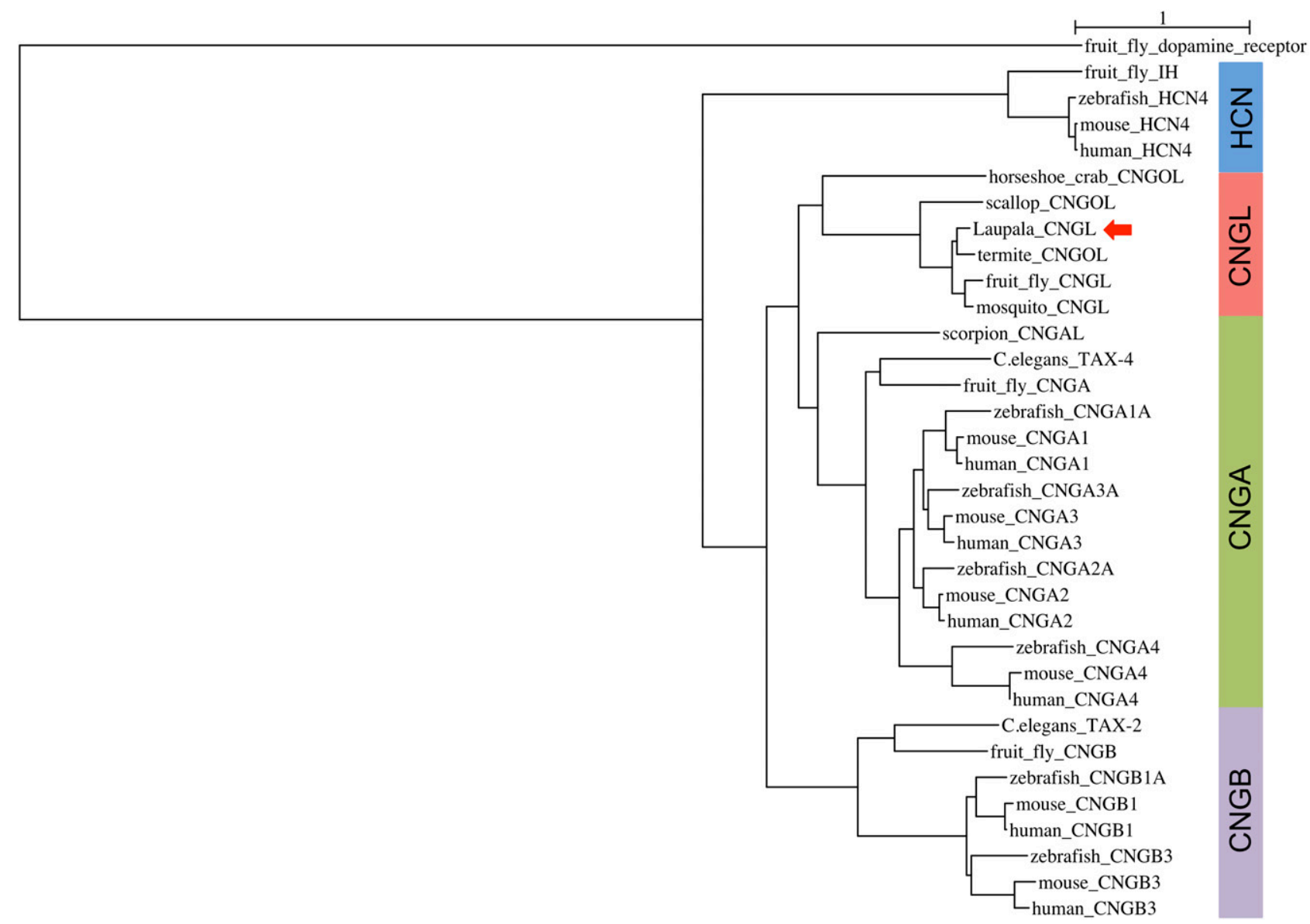

Figure 6 Maximum likelihood tree of amino acid sequences of conserved domains in cyclic nucleotide-gated ion channels subunit A1-4, B1, and B3, and hyperpolarization-activated cyclic nucleotide-gated ion channel subunit 4 from human (Homo sapiens), house mouse (Mus musculus), zebrafish (Danio rerio), and fruit fly (Drosophila melanogaster), homologous protein TAX-2 and TAX-4 in roundworm (Caenorhabditis elegans), as well as amino acid sequences of conserved domains in putative cyclic nucleotide-gated ion channel-like proteins identified in this study from scallop (Mizuhopecten yessoensis), horseshoe crab (Limulus polyphemus), scorpion (Centruroides sculpturatus), termite (Zootermopsis nevadensis), mosquito (Anopheles gambiae), fruit fly (Drosophila melanogaster), and cricket (Laupala kohalensis) using the LG model. Detailed information of the conserved domains represented in this phylogeny can be found in Table S6. The red arrow indicates the location of the putative Laupala CNGL protein.

we could not assign a linkage group were also unlikely to be the causal gene because the gene sequence and the $20-\mathrm{kb}$ flanking regions for each were completely homozygous between the two males we sequenced. The confidence interval region is also unlikely to contain duplicated copies of Drosophila song candidate genes because scaffolds within the confidence interval do not contain any significant blast hit against conserved domains in the 11 candidate genes. Therefore, it is unlikely that we missed mapping a Drosophila song candidate gene to LG5 because of the distant evolutionary relationship between crickets and flies. These results suggest that the causal gene underlying the major QTL on LG5 is likely a novel gene for song temporal pattern regulation.

To further probe for the causal gene, we conducted gene prediction and functional annotation of five focal scaffolds. The genomic region coverage estimation suggested that the linkage map has saturated the genomic region within the confidence interval of the major QTL (File S1). Thus, the causal gene is likely to be located on one of the focal scaffolds we annotated. We predicted 66 genes on scaffolds within the confidence interval. Twenty-eight of these 66 genes could not be annotated at an E-value cutoff of $1 \mathrm{E}-4$, although just 6 out of 66 could not be annotated at a relaxed E-value cutoff of 100 (Table S7). These unannotated genes were, nonetheless, retained as potential causal genes.

Of the 38 annotated genes, only four (Table S4; genes \#20, $\# 22$, \#23, and \#26) fall within our anticipated functional categories (ion transportation, neural modulation and development, and locomotion and muscle development) for the causal gene. Each of these genes resides on the scaffold with either the highest or the second highest LOD score. We acknowledge that by relying on SNPs and short indels in our 
1
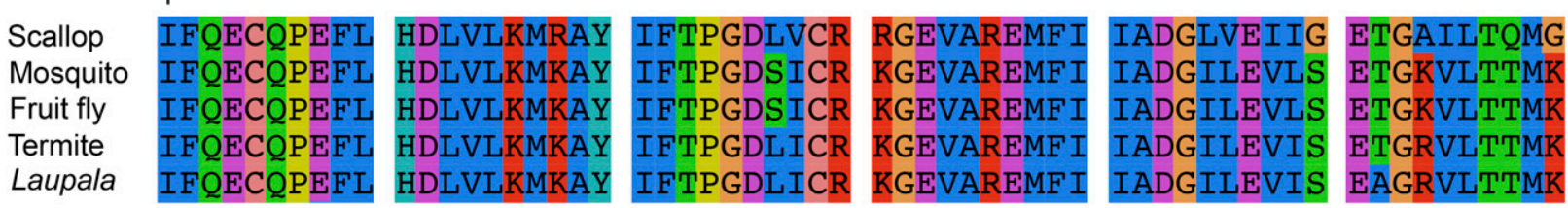

61
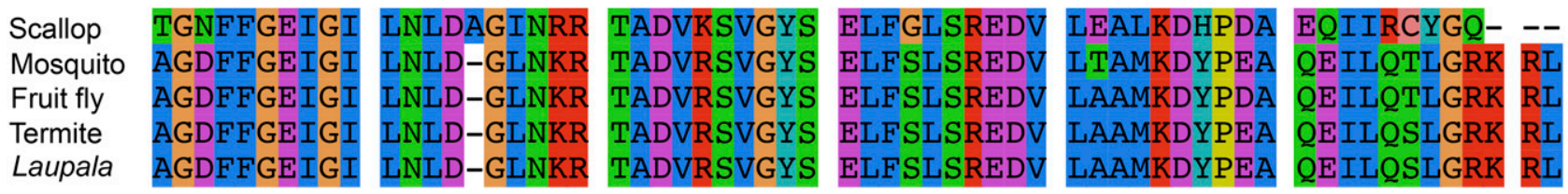

Figure 7 Multiple alignment of amino acid sequences of the cNMP binding domain, one of the domains used for building the tree in Figure 6, within putative homologous proteins of the cyclic nucleotide-gated ion channel-like protein in D. melanogaster. Protein sequence of the Laupala cNMP domain was translated from DNA sequence based on alignment to termite cNMP domain sequence in Exonerate (available in Figure S6).

evaluation of potential candidate genes, we may have missed other types of sequence variation in the predicted genes. The putative calcium release-activated calcium channel 1 gene (Orai1) and the putative signal peptide peptidase-like protein 3 gene (Sppl3), both on the highest LOD scaffold (S000353), have functions related to store-operated calcium entry into nerve and muscle cells, which is necessary for normal muscle function (Venkiteswaran and Hasan 2009; Prakriya and Lewis 2015). Specifically, in D. melanogaster, Orai has been shown to encode an integral component of the flight CPG and is required for both normal development and functioning of the flight circuit (Venkiteswaran and Hasan 2009; Pathak et al. 2015). In vertebrate cell lines, SPPL3 can enhance the association between Orai1 and the intracellular $\mathrm{Ca}^{2+}$ sensor gene STIM1 through its protease independent function (Makowski et al. 2015). These two genes, however, do not contain any WGS SNPs or indels. The putative synaptic vesicle 2-related protein gene (also known as SVOP), also located on the highest LOD scaffold, contains potential regulatory SNPs, has gene ontology annotation pertaining transmembrane transportation, and is expressed in developing nervous system (Janz et al. 1998; Logan et al. 2005). However, the specific function of this gene remains elusive and we could not find evidence directly linking this gene to the regulation of rhythmic behavior in either vertebrates or invertebrates.

Finally, a putative homolog of the Drosophila cyclic nucleotide-gated ion channel-like gene ( $\mathrm{Cngl}$ ) is the most promising candidate, the only one to fulfill all three criteria for a causal role in pulse rate variation. This gene resides on a scaffold immediately adjacent to the peak scaffold in 4C and 4E families. Members of the CNG gene family have been primarily described to function in signal transduction in the visual and olfactory sensory systems (Podda and Grassi 2014). However, the CNGL proteins have distinctive conserved domain architectures from all other CNG proteins (Table S6) and form a distinct clade from other CNG family channels (Figure 6). CNGL proteins are also longer, contain a unique section of $\mathrm{C}$ terminus sequence, and are expressed primarily in the brain, thoracic ganglia (wherein lies the CPG for pulse song in Drosophila; Clyne and Miesenböck 2008; von Philipsborn et al. 2011), and tubular fibers of muscles, as opposed to the photoreceptor, taste, and olfactory neurons, as in other CNG proteins (Miyazu et al. 2000). These distinctions suggest that Cngl may have functionally diverged from other CNG genes. In addition, members in the closely related hyperpolarization-activated cyclic nucleotide-gated ion channel (HCN) gene family (Figure 6) are known to modulate rhythmic behaviors across broad taxa and contexts, including rhythmic activity of the pyloric and gastric mills in crustaceans (Zhu et al. 2016), cardiac pacemaking in both invertebrates and vertebrates (Herrmann et al. 2015; Calabrese et al. 2016), and rhythmic activity of the respiratory network in mammals (Thoby-Brisson et al. 2000). CNG and HCN channels are structurally similar, share two conserved domains (Table S6), are both gated by intracellular cyclic nucleotides, and their genes exhibit a high level of sequence similarity, suggesting shared evolutionary history (Craven and Zagotta 2006). We therefore suggest that Cngl may have evolved to perform physiological functions in the CPGs of the wing muscles in insects.

We have not yet identified a transcript for the putative Cngl gene; future transcriptomes from both L. kohalensis and $L$. paranigra will offer crucial expression data to test functional differences of this gene between the two parental species.

Interestingly, although the QTL 4 confidence interval does not contain any $D$. melanogaster candidate genes, like cacophony or slowpoke, the most intriguing candidate for pulse rate difference in Laupala is also an ion channel gene. This result indicates that song temporal pattern evolution in crickets and flies may have taken genetically diverse, but functionally conserved routes. 
In summary, identifying targets of selection underlying behavioral divergence is crucial to understanding the early stage of speciation (Nosil and Schluter 2011). Toward this goal, genes identified from divergent lineages offer us more direct inference about genetic routes toward reproductive barriers in natural populations than candidate genes identified from mutational studies. Our annotation of the genomic region and identification of a promising candidate gene offers rare insights into the type of genetic variation, protein coding or regulatory, and its functional categorization underlying the evolution of a behavioral barrier in a rapidly speciating group of nonmodel organisms. Only with data from a diverse range of taxa can we begin to understand the general principles and mechanisms behind the evolution of reproductive barriers and rapid speciation.

\section{Acknowledgments}

We thank Ben Weaver, Alex Thomas, Eric Cole, and McKenzie Laws for assistance in cricket rearing; Cornell Institute for Genomic Diversity for advice on molecular procedures; and Aure Bombarely, Thomas Blankers, Linlin Zhang, and Cornell BioHPC laboratory for assistance in bioinformatics. Thomas Blankers and three anonymous reviewers provided comments that improved the manuscript. This project is funded by National Science Foundation grant 1257682 to K.L.S.

Author contributions: M.X. and K.L.S. designed and performed research. M.X. analyzed data. M.X. wrote the paper. K.L.S. edited the paper.

\section{Literature Cited}

Apweiler, R., A. Bairoch, C. H. Wu, W. C. Barker, B. Boeckmann et al., 2004 UniProt: the universal protein knowledgebase. Nucleic Acids Res. 32: D115-D119. https://doi.org/10.1093/nar/ gkh131

Aronesty, E., 2011 ea-utils: Command-Line Tools for Processing Biological Sequencing Data. Expression Analysis, Durham, NC.

Ashburner, M., C. A. Ball, J. A. Blake, D. Botstein, H. Butler et al., 2000 Gene ontology: tool for the unification of biology. Nat. Genet. 25: 25-29. https://doi.org/10.1038/75556

Bailey, N. W., P. Veltsos, Y. F. Tan, A. H. Millar, M. G. Ritchie et al., 2013 Tissue-specific transcriptomics in the field cricket Teleogryllus oceanicus. G3 (Bethesda) 3: 225-230. https:// doi.org/10.1534/g3.112.004341

Barkan, C. L., E. Zornik, and D. B. Kelley, 2017 Evolution of vocal patterns: tuning hindbrain circuits during species divergence. J. Exp. Biol. 220: 856-867.

Bay, R. A., M. E. Arnegard, G. L. Conte, J. Best, N. L. Bedford et al., 2017 Genetic coupling of female mate choice with polygenic ecological divergence facilitates stickleback speciation. Curr. Biol. 27: 3344-3349.e4. https://doi.org/10.1016/j.cub.2017. 09.037

Berdan, E. L., T. Blankers, I. Waurick, C. J. Mazzoni, and F. Mayer, 2016 A genes eye view of ontogeny: de novo assembly and profiling of the Gryllus rubens transcriptome. Mol. Ecol. Resour. 16: 1478-1490. https://doi.org/10.1111/1755-0998.12530
Blankers, T., K. P. Oh, A. Bombarely, and K. L. Shaw, 2018a The genomic architecture of a rapid island radiation: recombination rate variation, chromosome structure, and genome assembly of the Hawaiian cricket Laupala. Genetics 209: 1329-1344. https://doi.org/10.1534/genetics.118.300894

Blankers, T., K. P. Oh, and K. L. Shaw, 2018b The genetics of a behavioral speciation phenotype in an island system. Genes (Basel) 9: 346. https://doi.org/10.3390/genes9070346

Broman, K. W., H. Wu, G. A. Churchill, and Ś. Sen, 2003 R/qtl: QTL mapping in experimental crosses. Bioinformatics 19: 889890. https://doi.org/10.1093/bioinformatics/btg112

Calabrese, R. L., B. J. Norris, and A. Wenning, 2016 The neural control of heartbeat in invertebrates. Curr. Opin. Neurobiol. 41: 68-77. https://doi.org/10.1016/j.conb.2016.08.004

Camacho, C., G. Coulouris, V. Avagyan, N. Ma, J. Papadopoulos et al., 2009 BLAST+: architecture and applications. BMC Bioinformatics 10: 421. https://doi.org/10.1186/1471-2105-10421

Cantarel, B. L., I. Korf, S. M. Robb, G. Parra, E. Ross et al., 2008 MAKER: an easy-to-use annotation pipeline designed for emerging model organism genomes. Genome Res. 18: 188-196. https://doi.org/10.1101/gr.6743907

Chagnaud, B. P., and A. H. Bass, 2014 Vocal behavior and vocal central pattern generator organization diverge among toadfishes. Brain Behav. Evol. 84: 51-65.

Cingolani, P., A. Platts, L. Wang le, M. Coon, T. Nguyen et al., 2012 A program for annotating and predicting the effects of single nucleotide polymorphisms, SnpEff: SNPs in the genome of Drosophila melanogaster strain w1118; iso-2; iso-3. Fly (Austin) 6: 80-92. https://doi.org/10.4161/fly. 19695

Clyne, J. D., and G. Miesenböck, 2008 Sex-specific control and tuning of the pattern generator for courtship song inDrosophila. Cell 133: 354-363. https://doi.org/10.1016/ j.cell.2008.01.050

Coyne, J. A., and H. A. Orr, 2004 Speciation. Sinauer Associates, Sunderland, MA.

Craven, K. B., and W. N. Zagotta, 2006 CNG and HCN channels: two peas, one pod. Annu. Rev. Physiol. 68: 375-401. https:// doi.org/10.1146/annurev.physiol.68.040104.134728

Danecek, P., A. Auton, G. Abecasis, C. A. Albers, E. Banks et al., 2011 The variant call format and VCFtools. Bioinformatics 27: 2156-2158. https://doi.org/10.1093/bioinformatics/btr330

Danley, P. D., S. P. Mullen, F. Liu, V. Nene, J. Quackenbush et al., 2007 A cricket gene index: a genomic resource for studying neurobiology, speciation, and molecular evolution. BMC Genomics 8: 109. https://doi.org/10.1186/1471-21648-109

Ding, Y., A. Berrocal, T. Morita, K. D. Longden, and D. L. Stern, 2016 Natural courtship song variation caused by an intronic retroelement in an ion channel gene. Nature 536: 329-332. https://doi.org/10.1038/nature19093

Ellison, C. K., and K. L. Shaw, 2013 Additive genetic architecture underlying a rapidly evolving sexual signaling phenotype in the Hawaiian cricket genus Laupala. Behav. Genet. 43: 445-454. https://doi.org/10.1007/s10519-013-9601-2

Ellison, C. K., C. Wiley, and K. L. Shaw, 2011 The genetics of speciation: genes of small effect underlie sexual isolation in the Hawaiian cricket Laupala. J. Evol. Biol. 24: 1110-1119. https://doi.org/10.1111/j.1420-9101.2011.02244.x

Elshire, R. J., J. C. Glaubitz, Q. Sun, J. A. Poland, K. Kawamoto et al., 2011 A robust, simple genotyping-by-sequencing (GBS) approach for high diversity species. PLoS One 6: e19379. https://doi.org/10.1371/journal.pone.0019379

Fedotov, S. A., J. V. Bragina, N. G. Besedina, L. V. Danilenkova, E. A. Kamysheva et al., 2014 The effect of neurospecific knockdown of candidate genes for locomotor behavior and sound 
production in Drosophila melanogaster. Fly (Austin) 8: 176-187. https://doi.org/10.4161/19336934.2014.983389

Fedotov, S. A., J. V. Bragina, N. G. Besedina, L. V. Danilenkova, E. A. Kamysheva et al., 2018 Gene CG15630 (fipi) is involved in regulation of the interpulse interval in Drosophila courtship song. J. Neurogenet. 32: 15-26. https://doi.org/10.1080/ 01677063.2017.1405000

Finn, R. D., T. K. Attwood, P. C. Babbitt, A. Bateman, P. Bork et al., 2016 InterPro in 2017-beyond protein family and domain annotations. Nucleic Acids Res. 45: D190-D199. https:// doi.org/10.1093/nar/gkw1107

Garrison, E., 2012 Vcflib: A C++ library for parsing and manipulating VCF files. GitHub https://github.com/ekg/vcflib.

Garrison, E., and G. Marth, 2012 Haplotype-based variant detection from short-read sequencing. arXiv preprint arXiv.

Gerhardt, H. C., and F. Huber, 2002 Acoustic Communication in Insects and Anurans: Common Problems and Diverse Solutions. University of Chicago Press, Chicago.

Gleason, J. M., 2005 Mutations and natural genetic variation in the courtship song of Drosophila. Behav. Genet. 35: 265-277. https://doi.org/10.1007/s10519-005-3219-y

Gleason, J. M., and M. G. Ritchie, 2004 Do quantitative trait loci (QTL) for a courtship song difference between Drosophila simulans and $D$. sechellia coincide with candidate genes and intraspecific QTL? Genetics 166: 1303-1311. https://doi.org/ 10.1534/genetics.166.3.1303

Gleason, J. M., J.-M. Jallon, J.-D. Rouault, and M. G. Ritchie, 2005 Quantitative trait loci for cuticular hydrocarbons associated with sexual isolation between Drosophila simulans and $D$. sechellia. Genetics 171: 1789-1798. https://doi.org/10.1534/ genetics.104.037937

Gleason, J. M., R. A. James, C. Wicker-Thomas, and M. G. Ritchie, 2009 Identification of quantitative trait loci function through analysis of multiple cuticular hydrocarbons differing between Drosophila simulans and Drosophila sechellia females. Heredity 103: 416-424. https://doi.org/10.1038/ hdy.2009.79

Götz, S., J. M. García-Gómez, J. Terol, T. D. Williams, S. H. Nagaraj et al., 2008 High-throughput functional annotation and data mining with the Blast2GO suite. Nucleic Acids Res. 36: 3420-3435. https://doi.org/10.1093/nar/ gkn176

Greenspan, R. J., and J. F. Ferveur, 2000 Courtship in Drosophila. Annu. Rev. Genet. 34: 205-232. https://doi.org/10.1146/ annurev.genet.34.1.205

Groot, A. T., T. Dekker, and D. G. Heckel, 2016 The genetic basis of pheromone evolution in moths. Annu. Rev. Entomol. 61: 99-117. https://doi.org/10.1146/annurev-ento-010715023638

Guindon, S., and O. Gascuel, 2003 A simple, fast, and accurate algorithm to estimate large phylogenies by maximum likelihood. Syst. Biol. 52: 696-704. https://doi.org/10.1080/10635150390 235520

Hartbauer, M., and H. Römer, 2016 Rhythm generation and rhythm perception in insects: the evolution of synchronous choruses. Front. Neurosci. 10: 223. https://doi.org/10.3389/ fnins.2016.00223

Henry, C. S., M. L. M. Wells, and K. E. Holsinger, 2002 The inheritance of mating songs in two cryptic, sibling lacewing species (Neuroptera: Chrysopidae: Chrysoperla), pp. 269-289 in Genetics of Mate Choice: From Sexual Selection to Sexual Isolation. Springer, Dordrecht, The Netherlands.

Herrmann, S., S. Schnorr, and A. Ludwig, 2015 HCN channelsModulators of cardiac and neuronal excitability. Int. J. Mol. Sci. 16: 1429-1447.

Janz, R., K. Hofmann, and T. C. Südhof, 1998 SVOP, an evolutionarily conserved synaptic vesicle protein, suggests novel transport functions of synaptic vesicles. J. Neurosci. 18: 9269-9281. https://doi.org/10.1523/JNEUROSCI.18-22-09269. 1998

Kanehisa, M., Y. Sato, M. Kawashima, M. Furumichi, and M. Tanabe, 2016 KEGG as a reference resource for gene and protein annotation. Nucleic Acids Res. 44: D457-D462. https:// doi.org/10.1093/nar/gkv1070

Katz, P. S., 2016 Evolution of central pattern generators and rhythmic behaviours. Philos. Trans. R. Soc. Lond. B Biol. Sci. 371: 20150057. https://doi.org/10.1098/rstb.2015.0057

Korf, I., 2004 Gene finding in novel genomes. BMC Bioinformatics 5: 59. https://doi.org/10.1186/1471-2105-5-59

Kronforst, M. R., L. G. Young, D. D. Kapan, C. McNeely, R. J. O’Neill et al., 2006 Linkage of butterfly mate preference and wing color preference cue at the genomic location of wingless. Proc. Natl. Acad. Sci. USA 103: 6575-6580. https://doi.org/10.1073/ pnas.0509685103

Lamichhaney, S., F. Han, M. T. Webster, L. Andersson, B. R. Grant et al., 2018 Rapid hybrid speciation in Darwin's finches. Science 359: 224-228. https://doi.org/10.1126/science. aao4593

Langmead, B., and S. L. Salzberg, 2012 Fast gapped-read alignment with Bowtie 2. Nat. Methods 9: 357-359. https://doi.org/ 10.1038/nmeth.1923

Lassance, J.-M., A. T. Groot, M. A. Liénard, B. Antony, C. Borgwardt et al., 2010 Allelic variation in a fatty-acyl reductase gene causes divergence in moth sex pheromones. Nature 466: 486489. https://doi.org/10.1038/nature09058

Lassance, J.-M., M. A. Liénard, B. Antony, S. Qian, T. Fujii et al., 2013 Functional consequences of sequence variation in the pheromone biosynthetic gene pgFAR for Ostrinia moths. Proc. Natl. Acad. Sci. USA 110: 3967-3972. https://doi.org/10.1073/ pnas. 1208706110

Limousin, D., R. Streiff, B. Courtois, V. Dupuy, S. Alem et al., 2012 Genetic architecture of sexual selection: QTL mapping of male song and female receiver traits in an acoustic moth. PLoS One 7: e44554. https://doi.org/10.1371/journal.pone. 0044554

Logan, M. A., M. R. Steele, and M. L. Vetter, 2005 Expression of synaptic vesicle two-related protein SVOP in the developing nervous system of Xenopus laevis. Dev. Dyn. 234: 802-807. https://doi.org/10.1002/dvdy.20618

Makowski, S. L., Z. Wang, and J. L. Pomerantz, 2015 A proteaseindependent function for SPPL3 in NFAT activation. Mol. Cell. Biol. 35: 451-467. https://doi.org/10.1128/MCB.01124-14

Marchler-Bauer, A., M. K. Derbyshire, N. R. Gonzales, S. Lu, F. Chitsaz et al., 2015 CDD: NCBI's conserved domain database. Nucleic Acids Res. 43: D222-D226. https://doi.org/10.1093/ nar/gku1221

Mendelson, T. C., 2003 Sexual isolation evolves faster than hybrid inviability in a diverse and sexually dimorphic genus of fish (Percidae: Etheostoma). Evolution 57: 317-327. https:// doi.org/10.1111/j.0014-3820.2003.tb00266.x

Mendelson, T. C., and K. L. Shaw, 2002 Genetic and behavioral components of the cryptic species boundary between Laupala cerasina and L. kohalensis (Orthoptera: Gryllidae), pp. 301-310 in Genetics of Mate Choice: From Sexual Selection to Sexual Isolation. Springer, Dordrecht, The Netherlands.

Mendelson, T. C., and K. L. Shaw, 2005 Sexual behaviour: rapid speciation in an arthropod. Nature 433: 375-376. https:// doi.org/10.1038/433375a

Merrill, R. M., B. Van Schooten, J. A. Scott, and C. D. Jiggins, 2011 Pervasive genetic associations between traits causing reproductive isolation in Heliconius butterflies. Proc. R. Soc. Lond. B Biol. Sci. 278: 511-518. https://doi.org/10.1098/ rspb. 2010.1493 
Miyazu, M., T. Tanimura, and M. Sokabe, 2000 Molecular cloning and characterization of a putative cyclic nucleotide-gated channel from Drosophila melanogaster. Insect Mol. Biol. 9: 283-292. https://doi.org/10.1046/j.1365-2583.2000. 00186.x

Momigliano, P., H. Jokinen, A. Fraimout, A.-B. Florin, A. Norkko et al., 2017 Extraordinarily rapid speciation in a marine fish. Proc. Natl. Acad. Sci. USA 114: 6074-6079. https://doi.org/ 10.1073/pnas.1615109114

Mowles, S. L., M. Jennions, and P. R. Backwell, 2017 Multimodal communication in courting fiddler crabs reveals male performance capacities. R. Soc. Open Sci. 4: 161093. https:// doi.org/10.1098/rsos.161093

Niehuis, O., J. Büllesbach, A. K. Judson, T. Schmitt, and J. Gadau, 2011 Genetics of cuticular hydrocarbon differences between males of the parasitoid wasps Nasonia giraulti and Nasonia vitripennis. Heredity (Edinb) 107: 61-70. https://doi.org/ 10.1038/hdy.2010.157

Nosil, P., and D. Schluter, 2011 The genes underlying the process of speciation. Trends Ecol. Evol. 26: 160-167. https://doi.org/ 10.1016/j.tree.2011.01.001

Oh, K. P., G. L. Conte, and K. L. Shaw, 2013 Founder effects and the evolution of asymmetrical sexual isolation in a rapidlyspeciating clade. Curr. Zool. 59: 230-238. https://doi.org/ 10.1093/czoolo/59.2.230

Oh, K. P., and K. L. Shaw, 2013 Multivariate sexual selection in a rapidly evolving speciation phenotype. Proc. R. Soc. Lond. B Biol. Sci. 280: 20130482.

Otte, D., 1994 The Crickets of Hawaii: Origin, Systematics and Evolution. The Orthopterists' Society, Philadelphia.

Pathak, T., T. Agrawal, S. Richhariya, S. Sadaf, and G. Hasan, 2015 Store-operated calcium entry through Orai is required for transcriptional maturation of the flight circuit in Drosophila. J. Neurosci. 35: 13784-13799. https://doi.org/ 10.1523/JNEUROSCI.1680-15.2015

Podda, M. V., and C. Grassi, 2014 New perspectives in cyclic nucleotide-mediated functions in the CNS: the emerging role of cyclic nucleotide-gated (CNG) channels. Pflügers Arch. 466: 1241-1257.

Prakriya, M., and R. S. Lewis, 2015 Store-operated calcium channels. Physiol. Rev. 95: 1383-1436. https://doi.org/10.1152/ physrev.00057.2003

Rundus, A. S., R. D. Santer, and E. A. Hebets, 2010 Multimodal courtship efficacy of Schizocosa retrorsa wolf spiders: implications of an additional signal modality. Behav. Ecol. 21: 701-707. https:// doi.org/10.1093/beheco/arq042

Sæther, S. A., G.-P. Sætre, T. Borge, C. Wiley, N. Svedin et al., 2007 Sex chromosome-linked species recognition and evolution of reproductive isolation in flycatchers. Science 318: 95-97. https://doi.org/10.1126/science.1141506

Saldamando, C., S. Miyaguchi, H. Tatsuta, H. Kishino, J. Bridle et al., 2005 Inheritance of song and stridulatory peg number divergence between Chorthippus brunneus and C. jacobsi, two naturally hybridizing grasshopper species (Orthoptera: Acrididae). J. Evol. Biol. 18: 703-712. https://doi.org/10.1111/j.14209101.2004.00838.x

Sánchez-Guillén, R. A., A. Córdoba-Aguilar, A. Cordero-Rivera, and M. Wellenreuther, 2014 Rapid evolution of prezygotic barriers in non-territorial damselflies. Biol. J. Linn. Soc. Lond. 113: 485496. https://doi.org/10.1111/bij.12347

Schöneich, S., and B. Hedwig, 2017 Neurons and networks underlying singing behaviour, pp. 141-153 in The Cricket as a Model Organism: Development, Regeneration, and Behavior. Springer, Tokyo.

Seehausen, O., Y. Terai, I. S. Magalhaes, K. L. Carleton, H. D. Mrosso et al., 2008 Speciation through sensory drive in cichlid fish. Nature 455: 620-626. https://doi.org/10.1038/ nature 07285

Shaw, K. L., 1996 Polygenic inheritance of a behavioral phenotype: interspecific genetics of song in the Hawaiian cricket genus Laupala. Evolution 50: 256-266. https://doi.org/10.1111/j.15585646.1996.tb04489.x

Shaw, K. L., 2000 Interspecific genetics of mate recognition: inheritance of female acoustic preference in Hawaiian crickets. Evolution 54: 1303-1312. https://doi.org/10.1111/j.0014-3820. 2000.tb00563.x

Shaw, K. L., and D. P. Herlihy, 2000 Acoustic preference functions and song variability in the Hawaiian cricket Laupala cerasina. Proc. R. Soc. Lond. B Biol. Sci. 267: 577-584. https://doi.org/ 10.1098/rspb.2000.1040

Shaw, K. L., and S. P. Mullen, 2011 Genes vs. phenotypes in the study of speciation. Genetica 139: 649-661. https://doi.org/ 10.1007/s10709-011-9562-4

Shaw, K. L., Y. M. Parsons, and S. C. Lesnick, 2007 QTL analysis of a rapidly evolving speciation phenotype in the Hawaiian cricket Laupala. Mol. Ecol. 16: 2879-2892. https://doi.org/10.1111/ j.1365-294X.2007.03321.X

Slater, G. S., and E. Birney, 2005 Automated generation of heuristics for biological sequence comparison. BMC Bioinformatics 6: 31. https://doi.org/10.1186/1471-2105-6-31

Smith, M. E., K. K. Weller, B. Kynard, Y. Sato, and A. L. Godinho, 2018 Mating calls of three prochilodontid fish species from Brazil. Environ. Biol. Fishes 101: 327-339. https://doi.org/ 10.1007/s10641-017-0701-3

Stanke, M., and B. Morgenstern, 2005 AUGUSTUS: a web server for gene prediction in eukaryotes that allows user-defined constraints. Nucleic Acids Res. 33: W465-W467. https://doi.org/ 10.1093/nar/gki458

Starnberger, I., D. Preininger, and W. Hödl, 2014 The anuran vocal sac: a tool for multimodal signalling. Anim. Behav. 97: 281-288. https://doi.org/10.1016/j.anbehav.2014.07. 027

Thoby-Brisson, M., P. Telgkamp, and J. M. Ramirez, 2000 The role of the hyperpolarization-activated current in modulating rhythmic activity in the isolated respiratory network of mice. J. Neurosci. 20: 2994-3005. https://doi.org/10.1523/JNEUROSCI.20-08-02994.2000

Turner, T. L., P. M. Miller, and V. A. Cochrane, 2013 Combining genome-wide methods to investigate the genetic complexity of courtship song variation in Drosophila melanogaster. Mol. Biol. Evol. 30: 2113-2120. https://doi.org/10.1093/molbev/ mst111

Ullrich, R., P. Norton, and C. Scharff, 2016 Waltzing Taeniopygia: integration of courtship song and dance in the domesticated Australian zebra finch. Anim. Behav. 112: 285-300. https:// doi.org/10.1016/j.anbehav.2015.11.012

Van Ooijen, J. W., 2006 JoinMap 4, Software for the Calculation of Genetic Linkage Maps in Experimental Populations, p. 33. Kyazma $\mathrm{BV}$, Wageningen, The Netherlands.

Venkiteswaran, G., and G. Hasan, 2009 Intracellular $\mathrm{Ca}^{2+}$ signaling and store-operated $\mathrm{Ca}^{2+}$ entry are required in Drosophila neurons for flight. Proc. Natl. Acad. Sci. USA 106: 1032610331. https://doi.org/10.1073/pnas.0902982106

von Philipsborn, A. C., T. Liu, Y. Y. Jai, C. Masser, S. S. Bidaye et al., 2011 Neuronal control of Drosophila courtship song. Neuron 69: 509-522. https://doi.org/10.1016/j.neuron. 2011.01.011

Wiley, C., C. K. Ellison, and K. L. Shaw, 2012 Widespread genetic linkage of mating signals and preferences in the Hawaiian cricket Laupala. Proc. Biol. Sci. 279: 1203-1209. https:// doi.org/10.1098/rspb.2011.1740

Williams, M. A., A. G. Blouin, and M. A. F. Noor, 2001 Courtship songs of Drosophila pseudoobscura and D. persimilis. II. Genetics 
of species differences. Heredity 86: 68-77. https://doi.org/ 10.1046/j.1365-2540.2001.00811.x

Yamada, R., H. Kuba, T. M. Ishii, and H. Ohmori, 2005 Hyperpolarization-activated cyclic nucleotide-gated cation channels regulate auditory coincidence detection in nucleus laminaris of the chick. J. Neurosci. 25: 8867-8877. https:// doi.org/10.1523/JNEUROSCI.2541-05.2005

Zeng, V., B. Ewen-Campen, H. W. Horch, S. Roth, T. Mito et al., 2013 Developmental gene discovery in a hemimetabolous insect: de novo assembly and annotation of a transcriptome for the cricket Gryllus bimaculatus. PLoS One 8: e61479. https:// doi.org/10.1371/journal.pone.0061479

Zhu, L., A. I. Selverston, and J. Ayers, 2016 Role of $\mathrm{I}_{\mathrm{h}}$ in differentiating the dynamics of the gastric and pyloric neurons in the stomatogastric ganglion of the lobster, Homarus americanus. J. Neurophysiol. 115: 2434-2445. https://doi.org/10.1152/ jn.00737.2015

Communicating editor: C. Peichel 